G427

\section{IMPROVING DOCUMENTATION AND CLINICAL PRACTICE IN THE 6-8 WEEK BABY CHECK IN UK GENERAL PRACTICES}

${ }^{1}$ IE Wilson, ${ }^{2} \mathrm{~N}$ Kaushik, ${ }^{2} \mathrm{TL}$ Frank. 'Medicine, Wrightington, Wigan and Leigh NHS Foundation Trust, Wigan, UK; ${ }^{2}$ Bowland Medical Practice, Bowland Medical Practice, Manchester, UK

\subsection{6/archdischild-2015-308599.381}

Infants require a full physical examination within $72 \mathrm{~h}$ of birth and again between 6-8 weeks of age. NICE Clinical Guideline CG37 titled 'Routine postnatal care of women and their babies' specifies a 43-part list of expected examination points. We wanted to determine the proportion of infants attending for their 8-week baby check at a local General Practice, who had adequate documentation of examination findings in the electronic health records (EHR). The audit also aimed to determine if practice could be improved using an online template.

A retrospective audit assessing documentation of examination findings for the 8 -week check was performed on 20 patients attending the practice during a four-month period. $100 \%$ babies at the 6-8 week check should have all 43 criteria entered into the EHR. Each patient was given a score relating to the number of criteria documented. We then created a unique, easily identifiable '8-week baby check' electronic template for use with the EHR and doctors in the practice were trained in its use. Subsequent re-audit on a sample of 20 patients completed the audit cycle.

The initial audit showed overall percentage documentation was $22 \%$, well below the $100 \%$ standard. Best recorded were the red-reflex, heart sounds and hips, entered for 11 of 20 patients. 0 of 20 patients records commented on hands, feet, nose, ears, neck or presence of dysmorphism. 8 patients had only ' 8 -week check OK' entered into the EHR. Re-audit once the electronic template was in place showed a $73 \%$ improvement in documentation, with all 43 criteria being entered for at least $95 \%$ of the children.

The initial audit highlighted inadequate documentation of examination findings in the EHR at the 6-8 week newborn check. A well-designed intervention was shown to significantly improve practice thus maintaining medico-legally sound patient notes and optimising patient safety, as the template ensures comprehensive examinations are performed. With increasing shifts towards paperless advanced software systems, there are ample opportunities to improve the quality of care and documentation.

\section{G428(P) EXPOSURE TO ARTIFICIAL BRIGHT LIGHTS FROM MODERN ELECTRONIC MEDIA BEFORE BEDTIME MAY CONTRIBUTE TO SLEEP DIFFICULTIES IN CHILDREN/ YOUNG PEOPLE WITH / WITHOUT SPECIAL NEEDS- 3 PATIENT/ CARER SLEEP HYGIENE SURVEYS}

S Ozer. Child Development Centre, East and North Hertfordshire NHS Trust, Stevenage, UK

\subsection{6/archdischild-2015-308599.382}

Introduction Sleep onset difficulties may be worsened by exposure to artificial lights from modern electronic media with wavelengths similar to day light, potentially inhibiting the action of the sleep hormone melatonin responsible for sleep-wake cycle. A lot of current sleep hygiene advice for patients/families lack specific advice about the role of artificial lights in suppressing natural melatonin production.
Aims/objectives We examined pre bedtime/bedtime activities and sleep hygiene practices in special needs /non special needs children with sleep difficulties and explored level of pre-bedtime exposure to artificial lights from modern electronic media including computers, cell phones and digital television.

Methods Sleep hygiene surveys were conducted separately in carers of children with special needs-ADHD (6-18 years), preschool children with Autism Spectrum disorder (3-5 years) and a non special needs population of 500 primary school children in a socially deprived area from periods June 2013 to March 2014.

Results Children in both special needs and non-special needs populations kept some form of electronic media in their bedrooms $(26 \%)$ and played with these or watched television within $1 / 2-2 \mathrm{~h}(44 \%)$ before bedtime. Electronic device exposure was highest in the ADHD group (54\%). Majority (68\%) of carers in the ADHD cohort reported some improvement in sleep onset difficulties when they followed advice regarding switching electronic media off at least an hour before bedtime.

Conclusions There is an urgent need for Health and Educational Professionals to educate families about the role of modern electronic media/TV on the sleep cycle of children when conducting sleep education/hygiene.

There should be more public health awareness and research about the impact of modern electronic media and artificial lights on sleep in children with/without special needs and neurodevelopment disorders.

Outcome Following surveys, sleep tools have been developed to enable health and educational professionals conduct a more effective sleep interview and hygiene. Tools have been widely circulated to GPs, Health professionals and over 200 mental health Leads in schools. They have also been circulated on the National Child Trust UK website (NCTUK).

\section{G429(P) MELATONIN USE IN CHILDREN WITH SLEEP DIFFICULTIES: AN AUDIT OF PRESCRIPTION PRACTICES AMONGST EAST OF ENGLAND COMMUNITY PAEDIATRICIANS}

${ }^{1}$ ND Herberholz, ${ }^{2} S$ Ozer. ${ }^{1}$ Community Paediatrics, Cambridgeshire Community Services, Huntingdon, UK; ${ }^{2}$ Community Paediatrics, East and North Hertfordshire NHS Trust, Stevenage, UK

\subsection{6/archdischild-2015-308599.383}

Aims Disrupted sleep is commonly treated with melatonin, especially in children with Neurodevelopmental disorders. (BNF for Children 2014/2015). We surveyed melatonin prescription practices amongst Community Paediatricians in the East of England. Methods In May 2014 we sent out an online survey to all Community Paediatricians in the East of England.

Results 17 responses in total were received from all over the region.

All Paediatricians prescribe melatonin to children with Neurodevelopmental disorders, Neurodisability (73\%), Visual impairment (73\%), Intellectual disability (67\%) and to Children with Chronic Fatigue Syndrome (20\%) and behaviour difficulties (20\%). Sleep advice is given by all clinicians and promoted through leaflets (56\%), referral to Specialist Sleep clinics (38\%) and support from other professionals (School Nurses, 13\%, Occupational Therapists, 6\%, Intellectual Disability Services, 19\% and Family workers, 13\%). On average clinicians felt sleep hygiene should be tried for 3 months (range 1-6 months) before melatonin is started. Most (59\%) clinicians do not routinely 
assess impairment of function before prescribing melatonin. The main preparation of melatonin prescribed is tablets (94\%), followed by liquid (59\%) then capsules (41\%). Mostly (65\%) slow release medication is given. The minimum dose of melatonin prescribed is $2 \mathrm{mg}$ (range $0.5 \mathrm{mg}-3 \mathrm{mg}$ ), maximum dose range (4-12 mg).

Practice varies in how often children are reviewed. Some (35\%) review in the first 3-4 months, others 6 monthly (29\%). Most (94\%) clinicians offer at least yearly reviews. Routine trials off melatonin are offered by $65 \%$. On average children stay 26 months on melatonin before withdrawal (range 6-120 months). In comments Paediatrician gave views regarding the management of disrupted sleep, the need for good sleep hygiene support, which patient groups melatonin is best suited for and management of melatonin treatment.

Conclusions This survey has highlighted variability amongst Community Paediatricians in the East of England in certain areas of melatonin prescribing, possibly due to lack of uniform standards. With these results we are therefore creating a generic regional algorithm for initiating melatonin in children with disrupted sleep pattern which may form a platform for developing a melatonin prescription and sleep guideline for individual Organisations.

\section{G430(P) IS IT POSSIBLE TO HAVE HIGH STANDARDS PRACTICAL PATHWAY FOR AUTISM SPECTRUM DISORDER?}

A Tarhini. Community Paediatrics, Shropshire Community Health NHS Trust, Shrewsbury, UK

\subsection{6/archdischild-2015-308599.384}

Aims Public awareness of Autism Spectrum Disorder (ASD) is increasing, as well as the demand for assessment. The implementation of high standards and practical pathway for assessment and diagnosis can be a challenge for many trusts including our trust. The aim was to reconstruct the current pathway by the trust to produce a practical pathway for assessment and diagnosis of ASD that meet the high standards of NICE guidelines and following the DSM-V criteria.

Methods Firstly the strengths and weaknesses of the current pathway have been identified. Secondly set up the essential elements of the future pathway defined by DSM-V criteria and NICE guidelines for ASD assessment and diagnosis. An estimated time scale was calculated based on clinic and administration time required.

Results Three essential elements for the assessment have been identified: A) comprehensive meeting with paediatrician, B) multisource observational reports and C) direct assessment. It was possible to incorporate in each step some elements of the NICE guidelines and DSM-V criteria to cover all the essential elements and criteria. Finally, a set of recommendations and suggested pathway for the assessment and diagnosis of ASD was produced. The estimated time to make a definitive outcome about ASD is possible within $240 \mathrm{~min}$ of clinician's direct clinical and administration work.

See Figures 1 and 2

Conclusion It is essential for trusts to have a high standards and practical pathway for ASD diagnosis and assessment in line with NICE guidelines and DSM-V criteria. The current pathway was not satisfactory and a suggested practical pathway has been produced to meet the high standards of practice.

\section{Current Pathway}

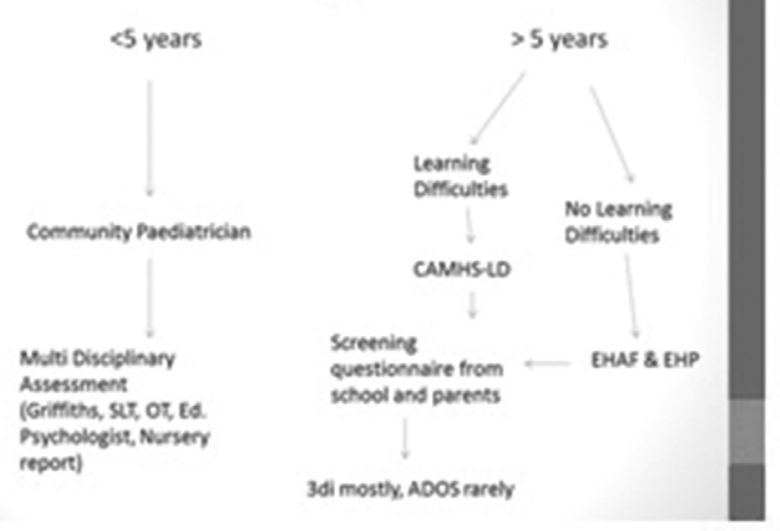

Abstract G430(P) Figure 1 Current pathway

\section{G431(P) AUDIT ON LOOKED AFTER CHILDREN AT RISK OF BLOOD-BORNE INFECTIONS}

P Mikrou, G Cropp, V Sadavarte. Child Development Centre, University Hospital of North Midlands, Stoke-on-Trent, UK

\subsection{6/archdischild-2015-308599.385}

Aims We aimed to assess whether Looked After Children (LAC) at risk of blood-borne infections (BBI) were identified, assessed, tested and referred as per the British Association for Adoption and Fostering (BAAF) guidance, in our specialist LAC service.

Methods Retrospective 12-monthly audit (June13-June14) of all children attending our specialist LAC clinic in Community Paediatrics in our University Teaching Hospital. Data collected from patient health reports, blood investigation results (including maternal antenatal infection screening) and information from Social Care on parental lifestyle. Data analysed using Microsoft Excel.

Results 212 children attended our specialist LAC clinic. 37 children $(17 \%)$ were identified as needing BBI screen. Out of these, only $22(60 \%)$ were screened. 8 out of 22 children $(36 \%)$ had a complete screen (Hepatitis $\mathrm{B} / \mathrm{C}$ and $\mathrm{HIV}$ ) with the remaining having a partial screen. 12 children had Hepatitis $\mathrm{C}$ positive mothers. Worryingly, only 8 of these 12 children (66\%) had BBI screen. There were no Hepatitis B or HIV positive mothers. Reasons for not having BBI screen were difficulties in obtaining consent, failure to identify those children at risk or to get the extended information about parental lifestyle and screening results. BBI screen revealed 2 children positive for Hepatitis C antibodies and appropriate follow-up was arranged. There were no children that had a BBI screen when that was not indicated.

Conclusion Our audit revealed a wide variation in practice as to which children have a BBI screen. We subsequently developed a protocol in the form of two flowcharts. These will be included in the LAC health assessment paperwork and aim to promote clarity and good clinical practice. As failure to obtain consent played an important hindering factor in getting our vulnerable population screened for BBI, we suggested that, when possible, consent is taken at the time of consultation. Improved communication and information sharing between Health and Social Care is essential. Finally, team education is greatly important and will 